\title{
EL AGUA, FUENTE PERMANENTE DE ENERGÍA
}

\author{
Luis Court M.
}

\section{INTRODUCCIÓN}

El agua es un factor determinante para la vida del ser humano y está presente, como un elemento básico en el desarrollo de la actividad económica y social del hombre.

La cantidad de agua disponible en las zonas continentales de la tierra, considerada en forma global, supera largamente las necesidades actuales de la humanidad, pero su distribución geográfica y en el tiempo no es uniforme y, en muchas oportunidades, tampoco guarda relación con la localización de los grandes centros urbanos, con las tierras aptas para el cultivo, con la ubicación de zonas industriales o mineras y, en general, con otras actividades de la sociedad humana. Es así como, a pesar de su relativa abundancia, en muchos países o regiones del globo terrestre se constituye en un claro factor limitante de las posibilidades de desarrollo.

Chile, dada sus características climáticas y geográficas, presenta en zonas importantes de su territorio claras limitaciones respecto a la disponibilidad del recurso agua.

El Norte Grande se caracteriza por una extrema escasez, debiéndose dar en él preferencia a los usos urbanos, mineros e industriales.

En el Norte Chico los recursos hidráulicos son más abundantes que en la región anterior, pero también bastante escasos. Ellos deben distribuirse en primera prioridad al consumo de sus poblaciones y a continuación a la minería y a la agricultura fundamentalmente. En este último rubro, en la medida que el recurso limitado lo permite.

Entre Aconcagua y Biobío los recursos de agua son más abundantes.

Esta zona se caracteriza por una agricultura de riego muy desarrollada y extensa, obteniéndose a través de ella una producción diversificada y de alta calidad. Además del uso del agua en la agricultura adquieren relevancia en esta zona los usos industriales y los de generación de hidroelectricidad.

Desde Biobío al sur los recursos de agua son abundantes, cobrando gran importancia en esta zona su utilización como fuente energética a través de la energía hidromecánica.

De acuerdo a lo expuesto, las zonas central y sur del país ofrecen posibilidades importantes de desarrollar hidroelectricidad, circunstancia que reviste mucha significación para Chile, pues su abastecimiento energético actual se realiza básicamente mediante hidrocarburos.

$\mathrm{Al}$ respecto, la situación del país en relación a los consumos de energía es aproximadamente la siguiente:

$\begin{array}{lr}\text { Petróleo } & 54 \% \\ \text { Gas Natural } & 11 \% \\ \text { Carbón } & 14 \% \\ \text { Hidroelectricidad } & 7 \% \\ \text { Leña y otros } & 14 \%\end{array}$


Según estas cifras, del orden de dos tercios del total de energía primaria proviene de los hidrocarburos, especialmente petróleo, que representa alrededor de un 54\% de dicho total. Considerando que solo una parte de este petróleo es de origen nacional (del orden de un 50\%), puede concluirse que nuestro abastecimiento energético es muy dependiente del exterior.

En relación a la energía hidromecánica, ella representa en la actualidad un aporte menor, de aproximadamente un 7\%, respecto al consumo.

Sin embargo, considerando que el agua constituye una posibilidad permanente de obtención de energía eléctrica, que ofrece claras ventajas sobre otras fuentes, por su carácter de recurso renovable, limpio y reutilizable, y porque nuestro país cuenta aún con importantes reservas hidroeléctricas ( $90 \%$ de su potencial), el recurso en Chile constituye una fuente energética de gran significación y que debe utilizarse a futuro cada vez más intensivamente.

\section{EL RECURSO AGUA EN CHILE}

El agua es un recurso natural que se presenta en una amplia gama de formas, tales como vapor, líquido (precipitaciones y escurrimientos superficiales o subterráneos), sólidos (nieve y hielo), etc.

La existencia de recursos de agua depende del clima, que es el que en último término provee toda el agua disponible en la superficie terrestre, fundamentalmente, a través de las precipitaciones.

Para los efectos de analizar el recurso agua del país y su utilización en hidroelectricidad es necesario, por lo tanto, referirse en primer término a las características climáticas del territorio y, como consecuencia de ellas, a los escurrimientos superficiales.

\section{Características climáticas del país}

La singular configuración geográfica de Chile condiciona la existencia de una gran diversidad de climas, desde el desértico por el norte hasta el polar por el sur.

Exceptuando la Antártica, Chile se extiende entre los $17^{\circ}$ y $53^{\circ}$ de latitud sur. En este desarrollo longitudinal está expuesto a variados efectos de la circulación general de la atmósfera terrestre que gobierna el clima continental. Hay regiones del país que se sitúan en la zona de los vientos alisios, otras en la de las calmas tropicales o cinturón de altas presiones subtropicales y otras en la de los vientos del oeste.

Particular importancia reviste la ubicación del país en la vertiente occidental de Sudamérica. En general, las cosas occidentales del continente americano son recorridas en una gran extensión por la corriente fría de Humboldt, lo que produce un efecto temperante en la atmósfera reforzado por la acción de los vientos, haciendo que las zonas australes tengan temperaturas más cálidas a las que debieran tener de acuerdo a la latitud y más frescas a medida que se avanza hacia la zona septentrional. Por esa razón 
las temperaturas a lo largo de Chile son menos extremas que en latitudes similares, a la vez que más uniformes al desplazarse en latitud. Así se observa, por ejemplo, que entre Arica y Navarino, con más de $4.000 \mathrm{~km}$ de distancia, hay una diferencia no mayor a $15^{\circ} \mathrm{C}$ en las temperaturas medias.

Otro factor importante en la determinación del clima del país es que ninguna región está demasiado lejos de la costa, razón por la cual la influencia del océano se hace sentir con mayor o menor intensidad en todo el territorio. Por este motivo en gran parte de él se presentan características de clima marítimo, siendo los veranos más frescos y los inviernos menos rigurosos que en las regiones de clima continental.

Dos accidentes del relieve, la cordillera de los Andes y de la Costa, juegan también un papel fundamental en la determinación del clima del país.

Debido a su magnitud, la cordillera de los Andes no solo influye en el clima local, sino también en extensas zonas alejadas a una gran distancia suya. Su efecto más significativo es el de constituir una gran barrera de norte a sur a lo largo del continente, que obstaculiza el paso de las perturbaciones atmosféricas que se mueven de oeste a este, dando origen a importantes precipitaciones orográficas responsables de los altos máximos pluviométricos que se observan en la vertiente occidental, en contraste con los mínimos existentes a la misma latitud en la vertiente oriental de los Andes. Otro aspecto es su rapidez para reaccionar frente al calentamiento y enfriamiento diarios, dando origen en las laderas andinas a intensos movimientos convectivos en la estación cálida, con las consiguientes consecuencias climáticas.

La cordillera de la Costa ejerce influencias similares que la de los Andes, pero de menor magnitud y extensión, por ser más baja que esta última. Su presencia se hace sentir con cierta intensidad en aquellas zonas donde el cordón montañoso alcanza anchuras o alturas máximas.

Un elemento importante en la determinación del clima del país es la ubicación del anticiclón del Pacífico.

Frente a la costa del Norte Chico, a una latitud que fluctúa entre los $30^{\circ}$ y $40^{\circ}$ aproximadamente y con una longitud de $100^{\circ}$ oeste, se encuentra la zona de altas presiones subtropicales (anticiclón del océano Pacífico), que forma parte del cinturón anticiclónico del hemisferio sur (Figura 1).

El anticiclón del Pacífico es una gran área en la que se producen las circulaciones generales de la atmósfera, típicas de los centros de alta presión, que hace sentir su efecto principalmente en la región oriental del mismo, produciendo condiciones de estabilidad atmosférica extrema. El aire sobre esta capa estable es muy seco y, como consecuencia de ello, el continente presenta alrededor de los $30^{\circ}$ de latitud sur una marcada carencia de precipitaciones.

El área anticiclonal se desplaza a lo largo del año en sentido norte-sur, alcanzando su posición más austral en los meses cálidos. La amplitud de la oscilación es del orden de los $10^{\circ}$. Su presencia en la costa norte es el factor dominante de la distribución de la presión atmosférica a lo largo del territorio nacional y un elemento determinante en el clima continental y el régimen de precipitaciones. 


\section{DISTRIBUCION DE PRESIONES ATMOSFERICAS A LO LARGO DE CHILE ISEOUN A. FONI)}

Destribución media do la presion en Enero

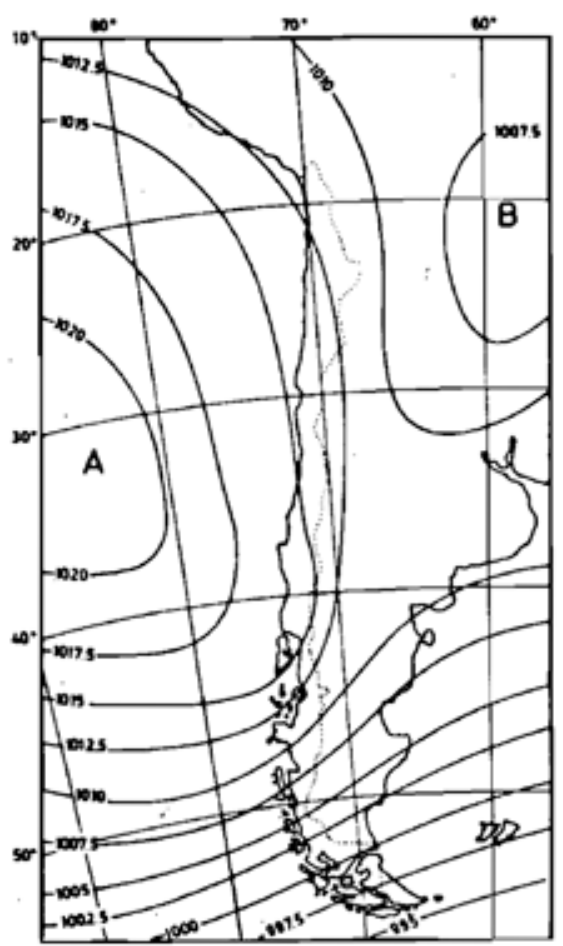

Distribución media de la presión en Julio

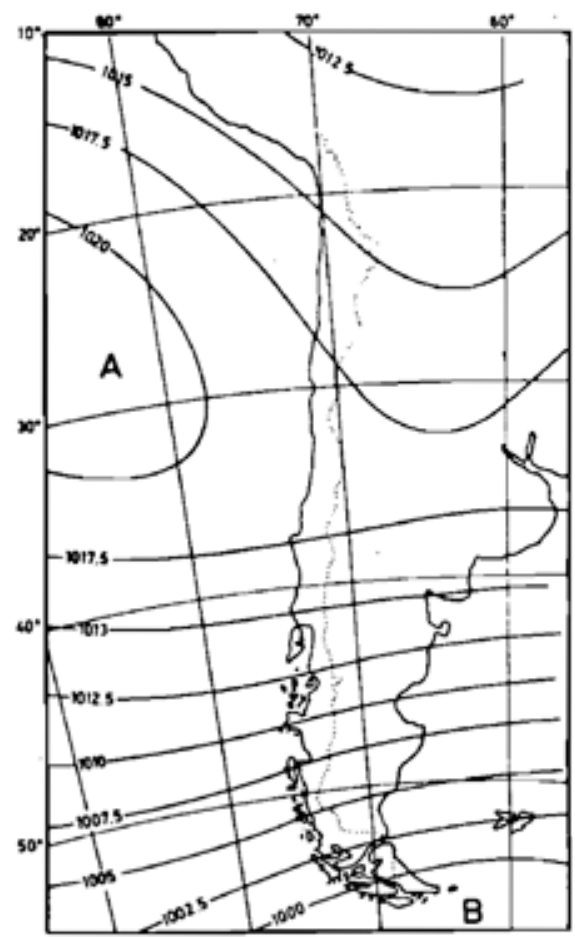

Figura 1

Desde el anticiclón hacia el polo sur se registra un descenso de la presión, de modo que mientras el norte del país está dentro de una zona de altas presiones, su extremo sur lo está en el área de bajas presiones circumpolares. La distribución de presiones mencionada es la causa de que en la mayor parte de la costa del país los vientos sean de dirección sur-oeste y sur.

Atendiendo a los aspectos enunciados se pueden establecer, a grandes rasgos, las siguientes regiones climáticas de Chile:

- Región de clima desértico

Aproximadamente entre la frontera norte y los $28^{\circ}$ de latitud sur, corresponde al Norte Grande. Está sometida a la acción de los vientos alisios y los efectos del cinturón de altas presiones subtropicales y, a excepción del extremo nororiental, las precipitaciones son escasas a tal punto de constituir una de las zonas más secas del orbe. 
Así, por ejemplo, mientras en Arica la precipitación anual promedio de un período de treinta años es de $1 \mathrm{~mm}$, en Iquique alcanza a 2,4 $\mathrm{mm}$, en Antofagasta a 4,9 mm y en Copiapó a 2,2 mm.

- Región de clima semidesértico

Corresponde al llamado Norte Chico y se ubica entre los $26^{\circ}$ y $31^{\circ}$ de latitud sur. Se distingue de la anterior en que durante el invierno se producen ocasionalmente precipitaciones de cierta importancia, cuando las perturbaciones atmosféricas procedentes del frente polar se extienden a las latitudes excepcionalmente más septentrionales de lo normal.

Mientras en los $18^{\circ}$ de latitud sur las precipitaciones anuales son del orden de 40 a $70 \mathrm{~mm}$, en los $31^{\circ}$ de latitud sur llegan a ser del orden de los $200 \mathrm{~mm}$.

- Región de clima mediterráneo

Situada entre los $31^{\circ}$ y $38^{\circ}$ de latitud sur, corresponde a la región del Valle Central. Se caracteriza por un verano seco y por un invierno en donde la acción de las perturbaciones del frente polar constituye un fenómeno más o menos activo todos los años. Cuanto más enérgica es esta acción más lluvioso y largo es el invierno.

Así, se tiene precipitaciones gradualmente mayores a medida que se avanza hacia el sur, variando de unos 200 a $350 \mathrm{~mm}$ entre los paralelos $32^{\circ}$ y $33^{\circ}$ a $1.500 \mathrm{~mm}$ en los $38^{\circ}$ de latitud sur.

- Región de clima lluvioso

Ubicada al sur del paralelo 38, se deja sentir la acción del frente polar durante todo el año, aunque a causa del desplazamiento estacional de este se produce una variación de las cantidades mensuales de precipitación de menor significación.

En esta región el régimen de precipitaciones es esencialmente variable.

Es así como en la zona de Puerto Montt las precipitaciones son del orden de los $2.000 \mathrm{~mm}$, alcanzando valores anuales tan altos como $8.000 \mathrm{~mm}$ en el área de los archipiélagos Wellington y Hannover, para luego decrecer hasta los 700 $\mathrm{mm}$ en la isla Navarino y $463 \mathrm{~mm}$ en la ciudad de Punta Arenas.

La Figura 2 muestra la distribución de la precipitación media anual entre los paralelos $30^{\circ}$ y $41^{\circ} 30^{\prime}$ de latitud sur siguiendo un perfil por el Valle Central del país. Se aprecia que las precipitaciones siguen una ley de variación creciente de norte a sur, hasta alcanzar un máximo entre los $39^{\circ}$ y $40^{\circ}$. Más al sur de los $40^{\circ}$ pareciera que las precipitaciones tienden a disminuir, pero en realidad en esta región del país no existe un gradiente pluviométrico tan uniforme, lo que impide formular una ley clara y única de variación en función de la latitud. 


\section{VARIACION DE LA PRECIPITACION MEDIA AINUAL EN CHILE \\ ENTRE LOS PARALELOS $30^{\circ}$ Y $41^{\circ} 30^{\circ}$ EN FUNCION DE LA LATITUD}

I VALORES OBTENIDOS A PARTIR DEL MAPA PLUVIOME TRICO DE CHILE SEGUN EL Dr. I. FONT I

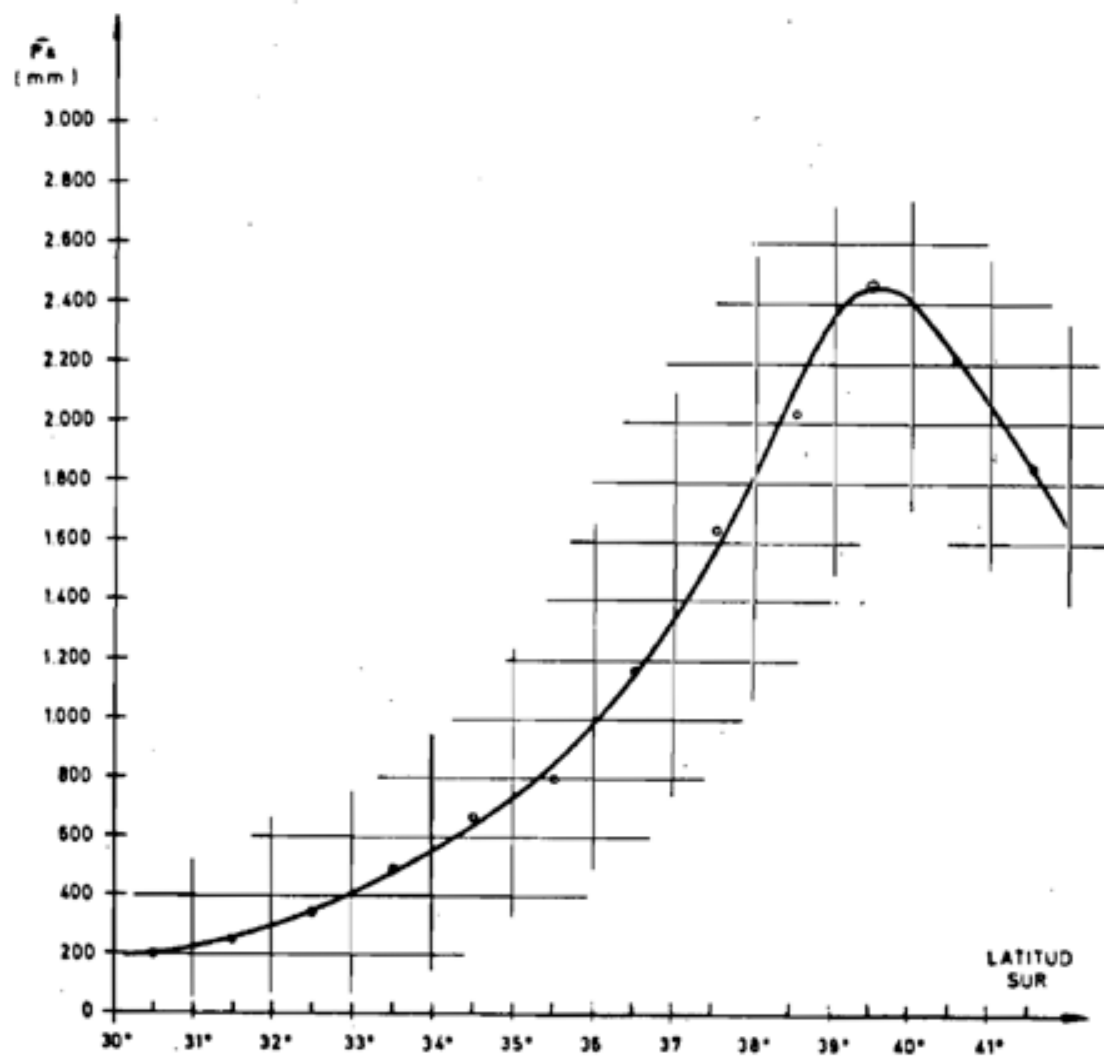

Figura 2

Dentro de este esquema general se presentan regiones de climas especiales. Uno de ellos es el clima de tundra que se encuentra principalmente en las regiones cordilleranas de la zona norte, adyacente a las regiones de clima de estepas, y con altitudes del orden de los 4.000 metros. Tales regiones se caracterizan por una intensa radiación sola en el día y fuerte pérdida de calor en la noche, lo que produce temperaturas de $10^{\circ}$ y $20^{\circ}$ bajo cero.

La complicada configuración geográfica de Chile y los factores analizados, dan lugar, además de las grandes zonas climáticas esquematizadas, a una gran variedad de climas que afectan zonas locales del territorio, pero que no influyen decisivamente en las características de los recursos de agua del país. 


\section{Características de los escurrimientos superficiales}

La existencia de escurrimientos superficiales está íntimamente ligada al régimen de precipitaciones, así como a condiciones de relieve, naturaleza geológica y vegetal de la región en que estos se producen. Siendo tan variables estos factores a lo largo del país, es lógico suponer que la distribución geográfica de los escurrimientos también lo sea.

Atendiendo a las características de los escurrimientos superficiales, pueden distinguirse tres zonas hidrográficas diferentes, que se indican a continuación:

a) Entre la frontera norte $y$ los $23^{\circ}$ de latitud sur

Se extiende una región de tipo "endorreico", que se caracteriza por cursos de agua intermitentes o mal desarrollados y que no logran llegar al océano.

Las precipitaciones son muy escasas en esta región y solo en el altiplano son de mayor significación. Sin embargo, es posible encontrar algunos recursos hidrológicos en forma de aguas corrientes en quebradas y ríos; otras veces como aguas retenidas en salares y lagunas, o también, como aguas subterráneas que suelen aflorar a la superficie, tomando el nombre de "aguadas".

A la carencia de precipitaciones en las mesetas y valles bajos de esta zona, se une el relieve especial que ella presenta, el cual no permite que las precipitaciones del altiplano contribuyan a formar escurrimientos superficiales de cierta magnitud. El relieve andino en esta región es, en general, suave, con numerosos niveles en forma de mesetas, a pesar de que se alcanzan alturas superiores a los 5.000 metros. La cordillera de los Andes presenta allí una serie de cordones montañosos que se extienden en dirección aproximada norte-sur, en los que se intercalan extensas mesetas favorables para el desarrollo de cuencas interiores de drenaje imperfecto, algunas de las cuales desaguan hacia el lado atlántico.

El desagüe imperfecto favorece la existencia de salares y lagunas de alguna magnitud. Siendo las precipitaciones del altiplano decrecientes hacia el sur, en el extremo norte de esta región se han desarrollado algunas lagunas importantes, como Chungará, que van desapareciendo hacia el sur para dar lugar a los salares, como el Ascotán, por ejemplo.

Los cauces superficiales que llegan al océano son una excepción. El desagüe se produce únicamente en algunos cursos importantes, con cabeceras extendidas hasta la región de precipitaciones significativas, capaces de haber profundizado suficientemente la cordillera de la Costa. Estos cauces existen principalmente en la parte septentrional de la provincia de Tarapacá y son los ríos Lluta, Azapa o San José y Camarones y la quebrada de Tana o Camiña.

Más al sur, en el extremo de esta región, se encuentra el río Loa, que es el más importante de los ríos del norte del país. Entre la quebrada de Tana y el río Loa no hay escurrimientos de importancia que logren llegar al mar. La mayoría corresponde a quebradas de débil alimentación y cauces modestos que desaparecen por infiltración.

Como las lluvias son de poca magnitud, los escurrimientos son débiles. 
La producción específica media de las cuencas de estos ríos no supera el litro por segundo por kilómetro cuadrado y su caudal medio anual no alcanza a los 5 $\mathrm{m} 3 / \mathrm{s}$. En cuanto al régimen de escurrimiento, los ríos de esta zona presentan avenidas ocasionales de gran caudal y corta duración en la época de precipitaciones en la cordillera, y caudales muy bajos o nulos en el resto del año.

\section{b) Entre los $23^{\circ}$ y $27^{\circ}$ de latitud sur}

Se desarrolla una región de tipo "arreica", cuya característica principal es que no tiene drenaje superficial.

Las características de relieve que se presentan en la región recién descrita desaparecen casi completamente al sur del río Loa. Fuera de la cordillera de Domeyko, que actúa como barrera para detener las aguas cordilleranas, no existen rasgos de relieve notables. Se presenta entonces una región caracterizada por la existencia de numerosas depresiones sin desagüe superficial, lo que da lugar a la formación de salares.

Los escurrimientos superficiales son de poca significación o inexistentes debido a la escasez de lluvias. Este factor, unido a las características del relieve, ha impedido la formación de cauces de caudal permanente. Mas al sur, entre Taltal y Copiapó, se encuentran algunos cauces pequeños, de caudal muy reducido, debido a que las precipitaciones son algo más abundantes.

c) Entre los $27^{\circ}$ y extremo austral

Se presenta una región de tipo "exorreico", que se caracteriza por cuencas con desagüe superficial al océano.

En esta región los escurrimientos superficiales son permanentes y de buen desarrollo, lo que es posible debido a que se presentan precipitaciones de cierta importancia, las cuales aumentan significativamente a medida que se avanza hacia el sur.

En general, en esta zona hasta Puerto Montt, la orografía presenta una "depresión intermedia" entre la cordillera de los Andes y de la Costa. Esta depresión da origen a los valles transversales en la parte norte (hasta el valle del río Maipo) y a un gran valle longitudinal desde el Maipo hacia el sur.

La cordillera de los Andes es de gran altura en la parte norte (con sus más altas cumbres en las zonas de Aconcagua y Maipo) disminuyendo gradualmente hacia el sur. Este relieve andino favorece la precipitación de tipo nival, lo que permite, como un gran embalse natural, su conservación hasta el verano en el sector norte y central de esta zona principalmente, contribuyendo a hacer más perdurable el efecto de las precipitaciones invernales. Con ello se favorece la existencia de cauces con drenaje permanente y de relativa importancia.

Las precipitaciones crecientes y de gran magnitud que se producen hacia el sur, posibilitan la formación de escurrimientos cada vez de mayor significación.

Los ríos comprendidos entre los $27^{\circ}$ y $32^{\circ}$ de latitud sur, vale decir, entre los ríos Copiapó y Choapa, son todavía de caudales limitados, sin sobrepasar los $5 \mathrm{~m} 3 / \mathrm{s}$ 
de caudal medio anual al abandonar la zona cordillerana. El caudal de estos ríos es muy uniforme a lo largo del año, presentando un leve aumento durante la época de deshielo (octubre a diciembre). La producción específica crece lentamente, pero sin sobrepasar los 10 litros por segundo y kilómetro cuadrado.

A partir de los $32^{\circ}$ y hasta los $36^{\circ}$ de latitud sur, o sea, entre los ríos Choapa y Maule, los ríos de cordillera tienen un régimen típico de deshielo en que el caudal aumenta significativamente a partir de octubre, alcanzando un caudal medio mensual máximo del orden de 2 a 2,5 veces el caudal medio anual en los meses de noviembre y diciembre. Después, comienza a decrecer hasta abril o mayo, para mantenerse más o menos constante en el resto del año con un caudal medio mensual del orden de 0,5 veces el caudal medio anual, valor alterado en mayor o menor grado por las crecidas invernales. En este sector, la producción específica crece rápidamente alcanzando en la cuenca del río Maule producciones específicas de 50 litros por segundo y kilómetro cuadrado.

Entre los ríos Nũule y Biobío, el régimen fluvial es de tipo mixto. Bajo la influencia creciente de las precipitaciones líquidas, los ríos muestran dos máximos, correspondiendo el primero al invierno y el segundo al período de deshielo, en el mes de noviembre o diciembre. La magnitud relativa de estos máximos depende directamente de las precipitaciones líquidas y sólidas caídas en la cuenca. Así, se tiene que en la zona cordillerana el máximo de deshielo es superior al de invierno y lo contrario sucede en el curso inferior del río. En la época pluvial los caudales mensuales suelen ser relativamente uniformes alcanzando valores un 10 a 20\% superiores al promedio anual; en cambio, en la época de deshielo, estos descienden hasta llegar a ser inferiores al $40 \%$ del caudal medio anual. Los caudales de estos ríos son de importancia, siempre crecientes hacia el sur, con producciones específicas variables entre los 50 y 70 litros por segundo y kilómetro cuadrado.

Entre el río Biobío y el río Puelo, los ríos presentan un régimen esencialmente pluvial. Durante los meses de abril a septiembre se concentra la mayor parte del volumen anual, correspondiendo a los meses de junio a agosto el máximo promedio mensual. A partir de estos meses el caudal empieza a disminuir, alcanzando su mínimo valor en marzo y abril. En algunos casos, el régimen presenta un leve aumento en el período de deshielo. Los caudales de estos ríos son muy abundantes, con producciones específicas que se incrementan desde los 70 litros por segundo y kilómetro cuadrado hasta un máximo de alrededor de 120 litros por segundo y kilómetro cuadrado.

Al sur del río Puelo los ríos se caracterizan por sus crecidas torrenciales y por las irregularidades de sus cursos. Siendo las precipitaciones en esta zona de gran magnitud, los escurrimientos superficiales también lo son. Casi todos los ríos de esta región nacen en la vertiente oriental de los Andes, con un desarrollo dilatado en las áreas trasandinas y como ríos bien formados cortan la cordillera de los Andes por valles desfiladeros típicos, presentando algunas veces grandes explanadas en el interior del sistema andino. La mayor parte presenta en su curso inferior rápidos y torrentes; algunos cruzan lagos que no modifican substancialmente al régimen del río. Los principales ríos de esta región son el Yelcho, el Palena, el Aisén, el Bravo, el Pascua y el Serrano. 
La situación presentada se puede sintetizar en el cuadro que se expone a continuación, en el cual se indica el caudal medio natural estimado en la desembocadura de los principales ríos del país.

\begin{tabular}{lcc}
\hline Rio & $\begin{array}{c}\text { Superficie total } \\
\text { de la cuenca }\left(\mathrm{km}^{2}\right)\end{array}$ & $\begin{array}{c}\text { Caudal medio natural } \\
\text { estimado } \\
\text { en su desemboca- } \\
\text { dura } \\
\left(\mathrm{m}^{3} / \mathrm{s}\right)\end{array}$ \\
\hline Loa & & 3 \\
Copiapó & 31925 & 3 \\
Huasco & 19190 & 4 \\
Elqui & 9456 & 11 \\
Limari & 9794 & 7 \\
Choapa & 11927 & 12 \\
Aconcagua & 8239 & 42 \\
Maipo & 7575 & 107 \\
Rapel & 14911 & 221 \\
Mataquito & 14503 & 151 \\
Maule & 5361 & 588 \\
ltata & 20965 & 214 \\
Biobio & 11633 & 761 \\
Imperial & 24262 & 658 \\
Tolten & 12464 & 516 \\
Valdivia & 8660 & 448 \\
Bueno & 11056 & 721 \\
Maullin & 15124 & 81 \\
Chamiza & 4738 & 45 \\
Petrohué & 725 & 277 \\
Puelo & 3036 & 685 \\
Aisén & 9001 & 600 \\
Baker & 11953 & 910 \\
Pascua & 27931 & 600 \\
\hline
\end{tabular}

\section{DESARROLLO DE LA HIDROELECTRICIDAD EN EL PAÍS}

El desarrollo del primer dínamo de servicio continuado, realizado por la empresa Siemens en 1866, la fabricación industrializada por la ampolleta incandescente con filamento de carbón, inventada por Tomás Alva Edison en 1879 y, luego, la evolución de los sistemas de transmisión en corriente alterna trifásica en el periodo que va desde 1880 a 1890, crearon las condiciones apropiadas para que la electricidad comenzase a ser la fuente de energía preferida, primero para el alumbrado público y posteriormente para el accionamiento de ferrocarriles y de motores industriales. 
La energía hidromecánica, como fuente de electricidad, comenzó su aplicación práctica en Chile ya en 1897, poco tiempo después de que la tecnología permitiera el transporte a distancia mediante líneas de transmisión. En ese año fue puesta en servicio la central Chivilingo que, mediante la utilización de una altura de caída de 110 metros y un grupo electrógeno de 500 KVA accionado por turbinas Pelton de marca Voith, comenzó a alimentar las incipientes demandas eléctricas de la Compañía Carbonífera de Lota, cuya mina se encontraba a $10 \mathrm{~km}$ de distancia de la central.

A esta primera central hidroeléctrica siguieron otras de mayor tamaño que, hasta el presente, se encuentran en operación regular, como son las centrales El Sauce de Valparaíso, de 1,4 MVA, y La Florida de Santiago, de 17 MVA, puestas en servicio en 1909 y 1913 respectivamente.

La primera guerra mundial interrumpió el desarrollo normal de los recursos hidroeléctricos, el que no fue retomado sino al término de esta. Tal hecho explica que no se hayan puesto nuevas centrales hidroeléctricas en servicio hasta después de 1920, en que entran en funcionamiento dos en el río Maipo, y que después pasarían a propiedad de CHILECTRA: Maitenes, de 24,4 MVA, puesta en operación en 1923, y Queltehues, de 40 MVA, que entró en explotación comercial en 1928.

En el decenio 1930-1940, los problemas de financiamiento derivados de la recesión mundial de 1929-1932, como también de las tarifas, que eran fijadas con prescindencia muchas veces de todo criterio técnico-económico, y la situación mundial incierta, provocada por la serie de hechos que finalmente condujeron al estallido de la segunda guerra mundial, restaron todo interés por realizar nuevas inversiones a las empresas privadas como CHILECTRA, CGEI, CONAFE y SAESA, cuyos capitales eran parcialmente de origen extranjero y que ya en aquella época eran las encargadas del suministro de servicio público en el país.

Como consecuencia de la incapacidad de las empresas de servicio público de satisfacer el crecimiento de las demandas eléctricas, las industrias importantes del país, para poder continuar su desarrollo, debieron construir sus propias centrales hidroeléctricas. Así fue como Cemento Melón puso en servicio su central Los Quilos, de 20 MVA, en el río Aconcagua, Carburo y Metalurgia (CARBOMET), la central Los Bajos, de 6 MVA, en el río Maipo, y la Compañía Manufacturera de Papeles y Cartones, sus centrales Puntilla, de 14 MVA, en el Maipo, y Carena, de 10, 4 MVA, que utiliza las aguas derivadas del río Mapocho mediante el canal Las Mercedes.

Con el fin de corregir la situación deficitaria en potencia eléctrica que se estaba generando en el país y que limitaba sus posibilidades de desarrollo, la CORFO creó en 1943 a la ENDESA, entregándole la responsabilidad de llevar a cabo prácticamente el mismo plan de electrificación del país que algunos años antes había propuesto al gobierno un grupo de ingenieros visionarios y auspiciado por el Instituto de Ingenieros de Chile.

La primera etapa del citado plan, que consultaba el abastecimiento de los principales centros de consumo del país mediante sistemas eléctricos locales alimentados por centrales hidroeléctricas, se realizó entre 1944 y 1952 con la construcción de las centrales Pilmaiquén en 1944, Sauzal y Abanico en 1948 y Los Molles en 1952. A continuación, se inició la segunda etapa de este plan, que contemplaba la interconexión 
de los diferentes sistemas eléctricos locales creados en la etapa anterior, con el fin de aprovechar los excedentes de cada zona y las ventajas de la complementación de los regímenes hidrológicos de los ríos situados en las zonas central y sur del país. Durante esta segunda etapa, que puede darse por finalizada en 1962, cuando quedó establecido el Sistema Interconectado Central entre La Serena y Puerto Montt, se construyeron las centrales Cipreses, Sauzalito y Pullinque, puestas en servicio respectivamente en 1955, 1959, 1962.

Finalmente, a partir de 1962 se ha estado ejecutando la tercera etapa del mencionado plan, que considera tanto el desarrollo de grandes centrales hidroeléctricas en el área del Sistema Interconectado Central, con el fin de aprovechar la economía de escala propia de estas obras, como el reforzamiento del sistema troncal de transmisión mediante la construcción de líneas de 220 a 500 KVA.

Durante esta tercera etapa, se han puesto en servicio las centrales Isla en 1963, Rapel en 1968, El Toro en 1973, Antuco en 1981 y Colbún y Machicura en 1985 (Figura 3).

Si bien todos estos aprovechamientos hidroeléctricos se han concentrado en la zona central del país, también ha habido una preocupación por desarrollar el potencial de las zonas extremas del país. Fruto de esta preocupación han sido la central Puerto Aisén, de 3 MW, puesta en servicio en 1962, y la Central Chapiquiña, ubicada al oriente de Arica, que se encuentra en operación desde 1967.

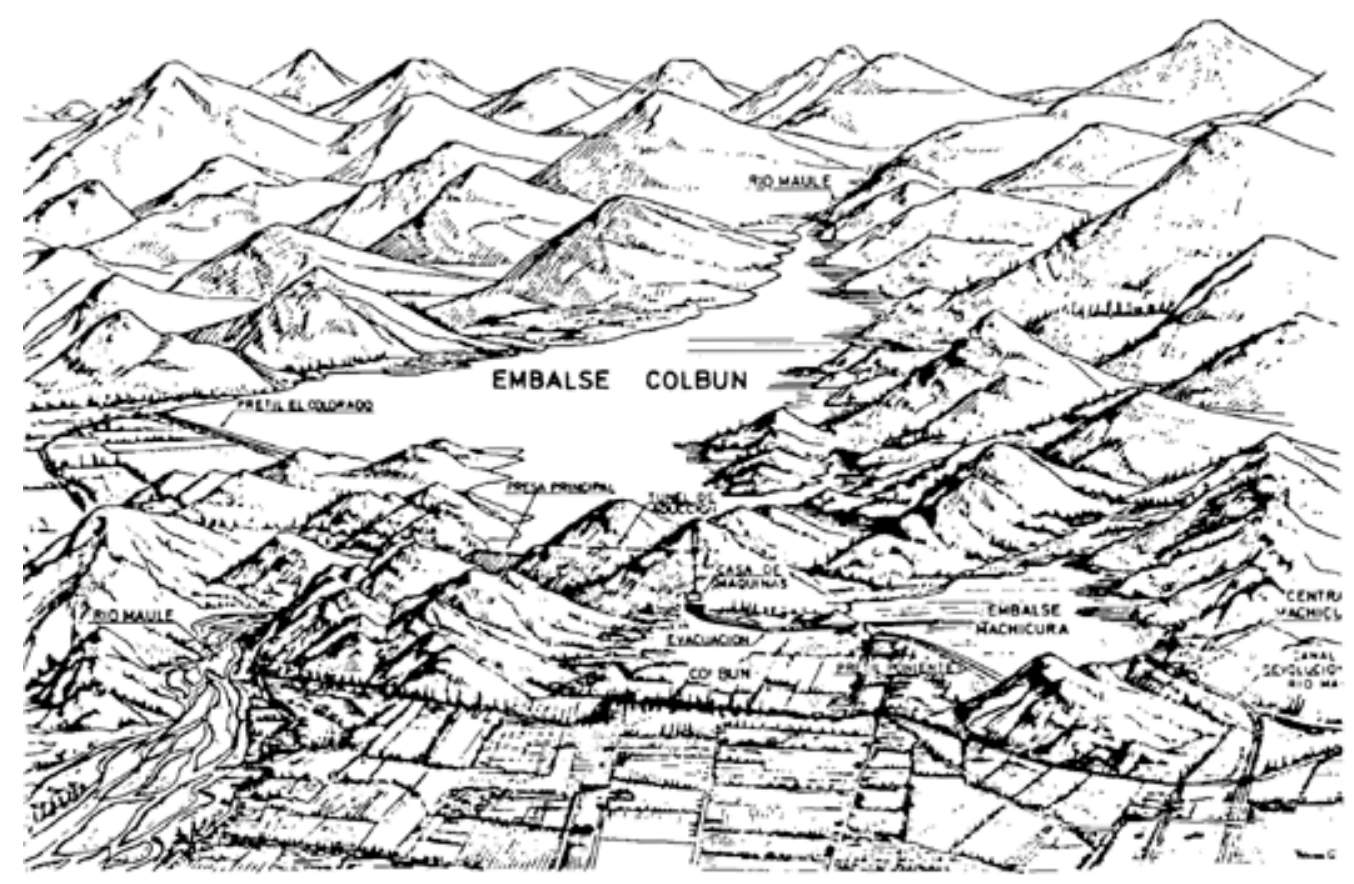

Figura 3 


\section{Potencial hidroeléctrico del país}

Chile es un país que cuenta con una topografía favorable para la existencia de recursos hidroenergéticos. Sin embargo, esta misma topografía que crea declives importantes en la dirección transversal este-oeste restringe la extensión de las cuencas hidrográficas de los ríos, limitando la magnitud de sus caudales. Es por ello que el potencial hidroenergético chileno resulta solo comparable con el correspondiente al promedio del continente sudamericano, existiendo varios países de este que lo superan al expresar este potencial ya sea como densidad por $\mathrm{km} 2$ o por habitante del país.

Los estudios realizados por la ENDESA en las últimas décadas han permitido establecer la magnitud del potencial técnicamente desarrollable en el país, entendiéndose por tal aquel que razonablemente puede suponerse que será explotado, aun cuando en las condiciones actuales no resulten económicamente competitivos. La magnitud de este potencial y su distribución en las zonas más características del país se indican en el cuadro A. De acuerdo con las cifras en él expuestas, los recursos hidroenergéticos nacionales permitirían la producción media anual de 127,6 TWh (miles de millones de KWh) y el desarrollo de una potencia del orden de 22.300 MW.

Es interesante observar que del citado potencial se encuentra desarrollado un total de 2.261 MW (incluyendo el complejo Colbún-Machicura), lo que representa apenas un 10\% del total. Como las centrales actualmente en operación tienen una producción media anual del orden de $11.100 \mathrm{GWh}$, se ve que, desde el punto de vista de producción de energía, apenas se está aprovechando el 8,7\% de la capacidad potencial del país.

En cuanto a la distribución geográfica de este potencial, puede notarse que, mayoritariamente, es aportado por las zonas centrales y austral del país, ya que la extensa zona que queda al norte de Los Vilos (zona norte en el cuadro A) no alcanza a proporcionar un $1 \%$ del total.

Conforme a estimaciones muy preliminares, basadas en la suposición del aumento del consumo eléctrico del país a una tasa constante de $6 \%$ y en una participación de energía térmica de un $20 \%$ del total en promedio, el desarrollo del potencial hidroenergético del país debería permitir la satisfacción del crecimiento de los consumos de los próximos cuarenta años, es decir hasta aproximadamente el decenio 2020-2030. Lógicamente que este pronóstico está sujeto no solo a que se cumplan los supuestos anteriores, sino que también a que todo el potencial técnico inventariado resulte económicamente explotable, lo que podría suceder en el futuro en caso de continuar encareciéndose las fuentes alternativas de energía eléctrica y de reducirse los costos de construcción de centrales mediante la introducción de nuevas técnicas y materiales de construcción.

\section{Visión a futuro del desarrollo del potencial hidroeléctrico del país}

El programa de obras para el abastecimiento futuro de las demandas eléctricas del país tiene por objetivo servir los incrementos de consumo que se vayan produciendo al mínimo costo actualizado que sea posible para el país. 


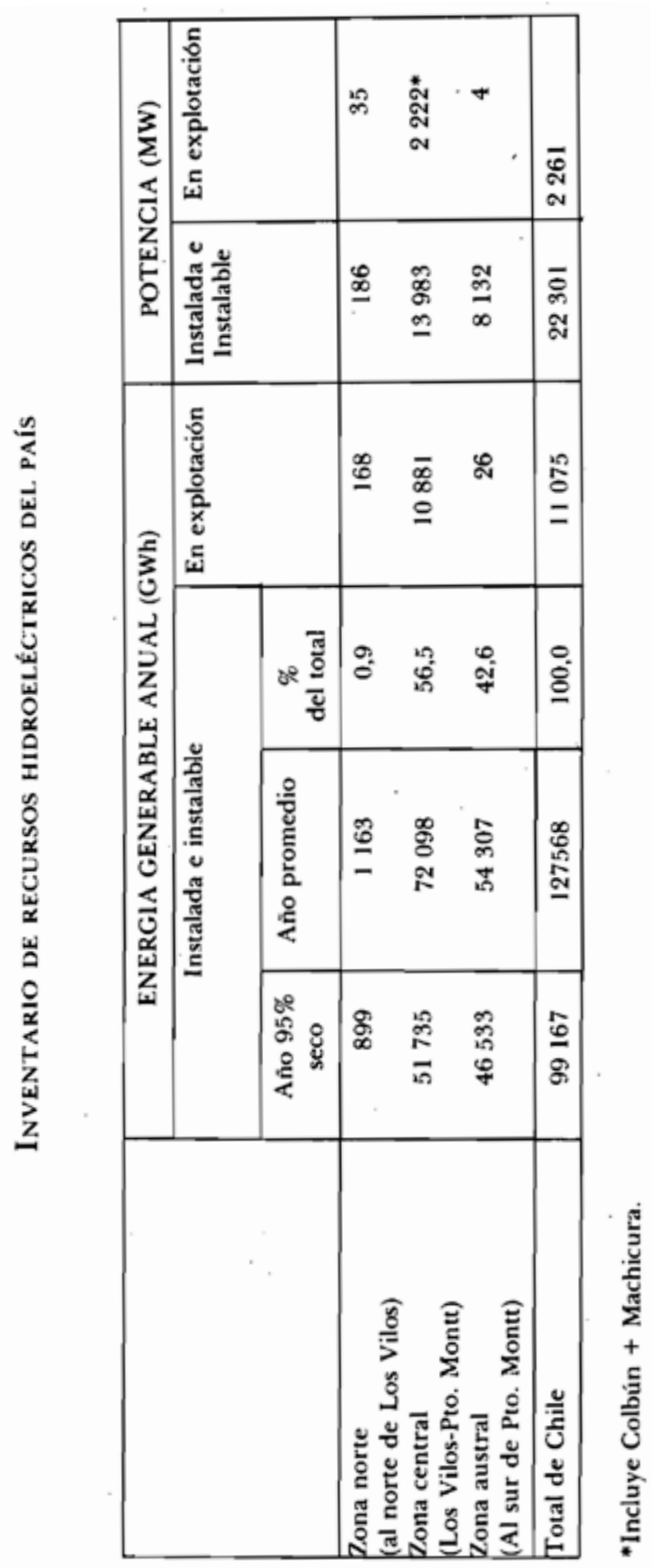


La aplicación del citado criterio de planificación conduce al desarrollo de aquellos recursos hidroeléctricos que, además de poseer características de generación favorables, requieran de inversiones comparativamente reducidas en obras de transmisión y de infraestructura.

Como consecuencia de lo anterior, en torno a las cuencas de los ríos Maule y Laja, se han ido conformando sendos núcleos de centrales hidroeléctricas que gravitan mayoritariamente en el abastecimiento del Sistema Interconectado Central.

En ellos, se concentra alrededor del $65 \%$ de la potencia hidroeléctrica instalada en este Sistema y se genera un promedio anual del orden de un $70 \%$ del total.

En el río Maule se encuentran en servicio las centrales Cipreses de 101,4 MW (1955), Isla de 68 MW (1963), Colbún de 400 MW (1985) y Machicura de 90 MW (1985). El aporte energético promedio anual del conjunto es de 4.200 GWh.

En el río Laja se encuentran en servicio Abanico de 136 MW (1948), El Toro de 400 MW (1973) y Antuco de 300 MW (1978). La generación promedio anual del conjunto es de aproximadamente $3.800 \mathrm{GWh}$.

Estos grandes aprovechamientos de los recursos hidroeléctricos concentrados en estas dos cuencas corresponde a la circunstancia que ellos están ubicados favorablemente respecto al muy extendido Sistema Interconectado, a que las obras de infraestructura de una determinada hoya pesan como inversión solo al comenzar el desarrollo de los recursos hidromecánicos, ya que a medida que se van incorporando nuevas centrales en la cuenca solo se necesita complementarlas, y sin necesidad de realizar grandes inversiones; a que el Sistema de Transmisión correspondiente en estos casos es más simple y permite realizar economías de escala, al concentrar en sistemas de alto o extra alto voltaje la transmisión de energía; a que el conocimiento del terreno en cuanto a geología, mecánica de suelos, clima, comportamiento de los ríos, etc. motivan precios de contratistas más competitivos, pues no deben asumir riesgos e imprevistos exagerados, si a través de las primeras centrales se van conociendo cada vez más claramente las características del terreno en el cual se construirán las centrales posteriores; a que los gastos de operación y mantención de las obras resultan muy bajos debido, justamente, a su concentración geográfica.

De acuerdo a lo expuesto, es un hecho real en nuestro país la tendencia a aprovechar en forma integral y relativamente sucesiva los recursos hidromecánicos de aquellas cuencas que están situadas estratégicamente respecto a los grandes centros consumidores y que ofrecen, además, posibilidades hidroeléctricas, técnica y económicamente favorables.

Actualmente, las posibilidades del río Laja se han utilizado casi en su totalidad. Las del río Maule se encuentran en una avanzada etapa de aprovechamiento, de modo que una vez que se construyan las centrales Pehuenche y Curillinque, en proyecto y estudio de factibilidad respectivamente, los recursos hidroeléctricos de esta cuenca estarían también desarrollados en su mayor parte.

Posteriormente, es posible visualizar como muy promisorio el aprovechamiento a mediano plazo del curso alto del río Biobío.

El plan de aprovechamiento hidroeléctrico del río Biobío contempla la construcción de seis centrales que operarían en serie hidráulica, aportando una potencia total 
de 2.900 MW y una generación media anual de 13.500 GWh. De estas centrales, las primeras en realizarse serían las de Ralco y Pangue, que en conjunto desarrollarían una potencia de 1.320 MW y una generación media anual de 6.230 GWh. Ambas centrales cuentan con proyectos de factibilidad muy avanzados, por lo que se espera que puedan encontrarse en servicio a mediados de la década 1990-2000. Las demás centrales, Llanquén (370 MW), Aguas Claras (660 MW) Huequecura (150 MW) y Quitramán (400 MW), de demostrarse su factibilidad económica, se construirían posiblemente a continuación.

En relación a otras cuencas con posibilidades favorables cabe mencionar a las del Alto Valdivia, donde se ubican los proyectos de Neltume y Choshuenco. Juntos, entregarían una potencia total de 550 MW con una generación media anual de 3.260 GWh y, posiblemente, se les construiría a fines de la década 1990-2000.

Los siguientes ríos, hacia el sur, que cuentan con recursos convenientes de explotar son el Chamiza, el Petrohué y el Puelo. El primero de los mencionados nace en el lago Chapo, que será la fuente que proveerá de agua a la central Canutillar. Esta ha sido programada para entrar en servicio en el año 1991. Con su potencia instalada de 130 MW estará en condiciones de generar unos 960 GWh de promedio anual, energía que será suficiente para cubrir los futuros consumos de toda la zona comprendida entre Temuco y Puerto Montt, y además entregar excedentes hacia el norte del país durante los primeros años de su funcionamiento.

En cada uno de los otros dos ríos se tienen proyectadas sendas centrales, de 450 MW la del Petrohué y de 1.250 MW la del Puelo. Esta última, posiblemente, será la de mayor potencia que se logrará construir en Chile.

Con la construcción de todas las centrales nombradas se habrá completado el desarrollo de los principales recursos que son de interés para el abastecimiento de toda la zona que servirá el Sistema Interconectado Central, que es posible que en un futuro llegue a abarcar desde Chiloé hasta el extremo norte del país (Figura 4).

Para continuar el desarrollo del potencial hidroeléctrico restante será preciso iniciar los proyectos de los ríos localizados en la región que queda al sur de Puerto Montt. Es probable que, para ello, sea necesario extender el sistema troncal de transmisión mediante un subsistema de corriente continua, ya que esta sería la forma más económica de transportar grandes bloques de energía a través de distancias considerables.

Después de completar el aprovechamiento de los recursos hidroenergéticos de la zona central se desarrollaría secuencialmente el potencial hidroeléctrico de los ríos Futaleufú, Palena, Aisén, Baker y Pascua, con lo que el aprovechamiento de los recursos más importantes que posee el país estaría en su mayor parte realizado. En esa época, posiblemente en el tercer decenio del próximo siglo (2020-2030), quedarían solo unos pocos recursos hidroeléctricos disponibles, que serían técnica y económicamente menos favorables, por lo que el país, para abastecer sus crecientes demandas, tendría que concentrar sus esfuerzos en nuevas fuentes de energía, como podrían ser el carbón, el calor de origen geotérmico o solar, los combustibles nucleares, como uranio, plutonio o torio, o bien, las reacciones termonucleares de fusión que se encuentran en la actualidad en etapa de experimentación, $\mathrm{u}$ otra fuente desconocida en el presente. 


\section{LOCALIZACION DE LAS PRINCIPALES FUENTES DE RECURSOS HIDROENERGETICOS DEL PAIS}

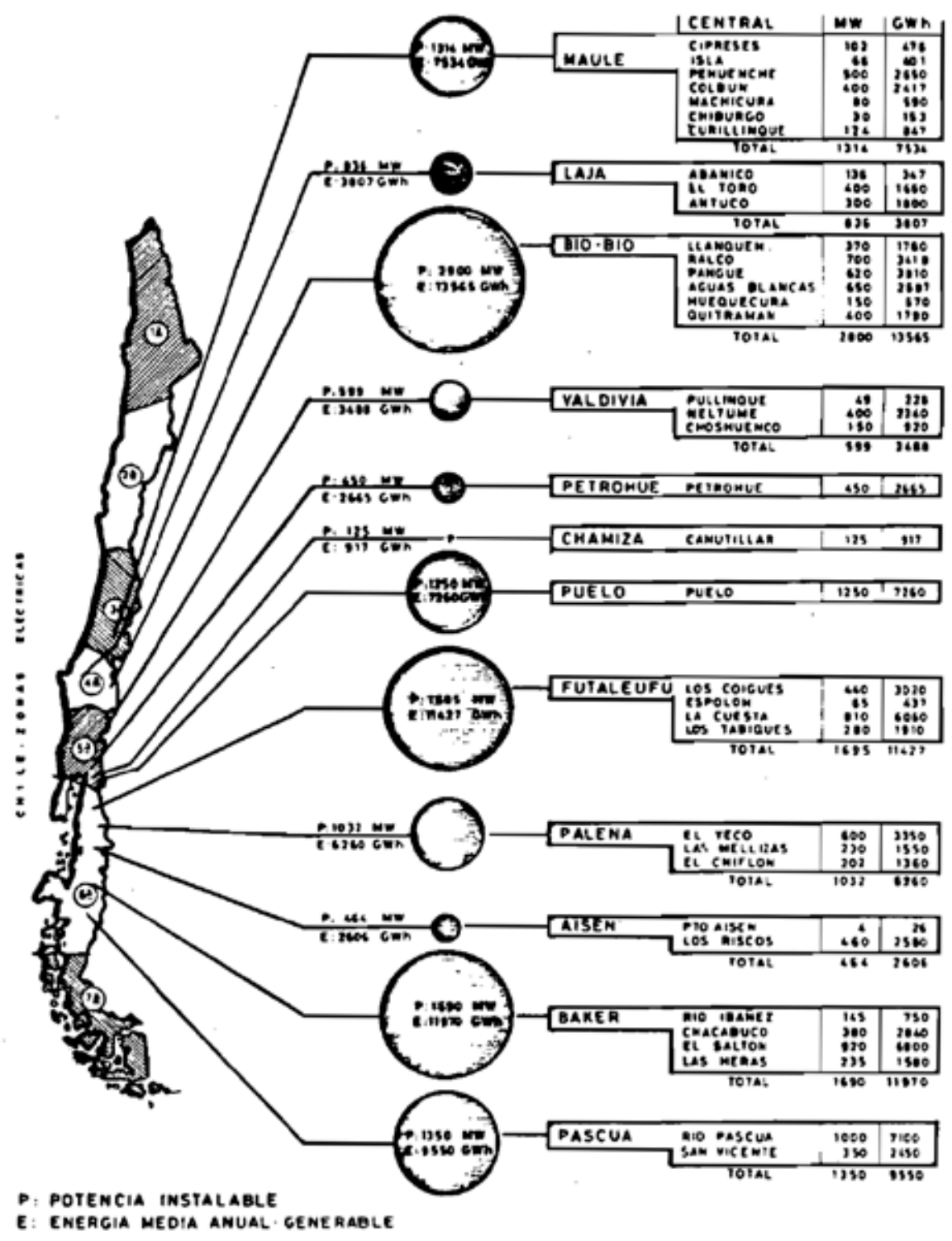

Figura 4

Todo lo expuesto anteriormente se refiere a la visión que se pueda dar respecto a la forma en que se van a explotar los recursos hidroeléctricos de gran magnitud, cuya generación se destinará al abastecimiento de los consumos de servicio público que se generarán principalmente en la zona central del país, servida por el Sistema Interconectado Central. Sin embargo, es posible prever que a futuro, además de las centrales de gran o 
mediana potencia instalada, se desarrollarán minicentrales hidroeléctricas, especialmente en la zona austral del país. En las Regiones X y XI, existen diversos pueblos aislados, tanto por las grandes distancias que los separan de las ciudades principales como por la mala calidad de sus caminos de acceso, que por esta razón cabe esperar que no se integren a ninguno de los sistemas eléctricos existentes, por lo menos por los próximos veinte a treinta años. Entre estos pueblos cabe mencionar a Río Negro, Hornopirén, Chaitén, Palena y Futaleufú, ubicados en Chiloé continental, a La Junta, Puyuhuapi y Puerto Cisnes, situados cerca o en el litoral del territorio que queda al norte de Aisén, y a Chile Chico, Guadal, Puerto Bertrand o Cochrane, localizados en los alrededores o cerca de las márgenes del lago General Carrera.

En todos los pueblos mencionados existen redes eléctricas de servicio público alimentadas por grupos diesel, proporcionando un suministro eléctrico de unas 5 horas diarias de promedio anual.

De acuerdo con los estudios realizados por CORFO y ENDESA, la forma más económica de dar un suministro eléctrico continuado en estos lugares es construyendo minicentrales hidroeléctricas en las cercanías de los pueblos que han de alimentar, y creando pequeños sistemas eléctricos en aquellos casos en que la distancia entre pueblos justifique su interconexión.

En concordancia con la conclusión anterior, se ha considerado la construcción de una serie de minicentrales ubicadas en las cercanías de los pueblos antes mencionados y que cumplen con los adecuados requisitos de seguridad de suministro y facilidad de desarrollo.

El programa respecto a estos recursos incluye, por el momento, la construcción de nueve minicentrales dentro de los próximos tres años, con lo que se espera resolver el problema del racionamiento del suministro eléctrico de los pueblos aislados de las Regiones X y XI.

\section{REFERENCIAS BIBLIOGRÁFICAS}

Almeyda A., E. y Sáez S., F., Recopilación de datos climáticos de Chile, Ministerio de Agricultura, 1958.

Asociación de Ingenieros, Los recursos de agua en Chile y su utilización en la generación de energía eléctrica, ENDESA, 1974.

La energía eléctrica en Chile, ENDESA, 1976.

Visión de la ENDESA, ENDESA, 1984.

Caudales medios mensuales retrospectivos, ENDESA, 1972.

Centro de Planeamiento, Estudio de la disponibilidad de recursos de agua en Chile, Facultad de Ciencias Físicas y Matemáticas, Universidad de Chile, Publicación 65-5/B, 1965.

Dirección General de Aguas, Caudales medios mensuales de los ríos de Chile, Vol. I, 1976.

Font, I., Factores que gobiernan el clima en Chile, ENDESA, 1963. 